\title{
TOLERANCIA A LA DESECACIÓN EN SEMILLAS DE TRES ORÍGENES GENÉTICOS DE CACAO (Theobroma cacao L.)
}

\author{
DESICCATION TOLERANCE IN SEEDS FROM THREE GENETIC ORIGINS OF COCOA \\ (Theobroma cacao L.)
}

\author{
Ma. Alma Rangel Fajardoํㅡㄹ Leobigildo Córdova Téllez ${ }^{1 \star}$, Alejandro P. López Andrade ${ }^{2}$, Adriana Delgado \\ Alvarado $^{3}$, Hilda A. Zavaleta Mancera ${ }^{3}$ y Ángel Villegas Monter ${ }^{1}$
}

\begin{abstract}
${ }^{1}$ Instituto de Recursos Genéticos y Productividad, Colegio de Postgraduados-Campus Montecillo. Km 36.5 Carretera México-Texcoco. 56230, Montecillo, Texcoco, Edo de México. ${ }^{2}$ Centro de Investigación Huimanguillo, Tabasco, Instituto Nacional de Investigaciones Forestales, Agrícola y Pecuaria. Km. 1 Carretera Humanguillo-Cárdenas. 86400, Huimanguillo, Tab. ${ }^{3}$ Instituto de Recursos Naturales, Colegio de Postgraduados-Campus Montecillo. Km 36.5 Carretera MéxicoTexcoco. 56230, Montecillo, Texcoco, Edo de México.
\end{abstract}

*Autor para correspondencia (lcordova@colpos.mx)

\section{RESUMEN}

Las semillas que no toleran la desecación (recalcitrantes), como las de Theobroma cacao L., mueren durante el secado, pero el contenido de humedad al cual mueren puede variar entre genotipos de la misma especie. Aquí se estudió la sensibilidad a la desecación de cinco genotipos de cacao de tres orígenes genéticos, y los cambios físicos en las semillas en la última etapa de su desarrollo. Frutos de 'Pound 7' y 'EET48' (Forasteros), 'UF667' y 'UF668' (Trinitarios) y “Carmelo" (Criollo) se cosecharon a los 5, 6 y 7 meses después de floración (mdf), y en la semilla se midió largo, ancho, espesor, contenido de humedad inicial y peso seco. Las semillas se secaron a 300, 200 y $150 \mathrm{~g} \mathrm{H}_{2} \mathrm{O} \mathrm{kg}^{-1}$ pf, y la humedad testigo fue la de una muestra recién cosechada. El efecto del secado se midió en la germinación y en la estructura de los ejes embrionarios. Los resultados indican que entre fechas de cosecha y genotipos la humedad de las semillas fluctuó de $529 \mathrm{~g} \mathrm{H}_{2} \mathrm{O} \mathrm{kg}^{-1} \mathrm{pf}$ ('EET 48') a $706 \mathrm{~g} \mathrm{H}_{2} \mathrm{O} \mathrm{kg}^{-1}$ pf ('Carmelo'). El peso seco incrementó en $2.5 \mathrm{~g}$ $(236 \%)$ de la cosecha uno $(5 \mathrm{mdf})$ a la tres $(7 \mathrm{mdf})$, que correspondió con aumentos de 0.6 y $4 \mathrm{~mm}$ en el ancho y espesor de la semilla. En semillas con la humedad al momento de cosecha, la germinación fue de $94 \%$. Esta tasa disminuyó en promedio, a 50 y $0 \%$ al reducir la humedad a 300 y $200 \mathrm{~g} \mathrm{H}_{2} \mathrm{O} \mathrm{kg}^{-1}$ pf. Los genotipos menos sensibles a la desecación de $300 \mathrm{~g} \mathrm{H}_{2} \mathrm{O}^{2-1}$ fueron los genotipos 'EET48' y 'UF667'. La desecación de la semilla modificó la estructura celular de los ejes embrionarios. Los genotipos 'Carmelo', 'Pound 7' (Forastero) y 'UF668' mostraron daños (encogimiento del citoplasma, pérdida de la afinidad de los ácidos nucleicos por la Safranina y pérdida de núcleos) en $\mathbf{5 0} \%$ de sus células embrionarias, cuando la humedad se redujo a 300 y a $200 \mathrm{~g} \mathrm{H}_{2} \mathrm{O} \mathrm{kg}^{-1}$ pf. El genotipo menos sensible fue 'UF668'. Se concluye que la sensibilidad a la desecación varía entre los genotipos de los tres orígenes genéticos a $300 \mathrm{~g} \mathrm{H}_{2} \mathrm{O} \mathrm{kg}^{-1}$, pero ninguno tolera el secado a 200 $\mathrm{g} \mathrm{H}_{2} \mathrm{O} \mathrm{kg}^{-1} \mathrm{pf}$.

Palabras clave: Theobroma cacao, contenido de humedad, grado de madurez, recalcitrancia, sensibilidad a desecación.

\section{SUMMARY}

Recalcitrant seeds such as Theobroma cacao L. die during the drying, but the moisture content at which dying takes place may vary among genotypes within the same species. In this research, desiccation sensitivity to drying was measured in five genotypes of cocoa from three genetic origins, and the physical changes of seeds were observed during the last stage of development. Fruits from 'Pound 7' and 'EET48' (Forasteros), 'UF667' and 'UF668' (hybrids) and 'Carmelo' (Criollo) were harvested at 5, 6 and 7 months after flowering (maf). Variables evaluated were: length, width, thickness, initial moisture content, and seed dry weight. Seeds were dried-down to 300,200 and $150 \mathrm{~g} \mathrm{H}_{2} \mathrm{O} \mathrm{kg}^{-1}$ fw, using fresh-seed samples as controls. The effect of seed drying at the three harvest dates was evaluated through germination rates and structural changes in the embryo axes, radicle apical meristems and caliptre. Results indicated that seed moisture content varied among harvests and genotypes, from $529 \mathrm{~g} \mathrm{H}_{2} \mathrm{O} \mathrm{kg}^{-1} \mathrm{fw}$ ('EET48') to $706 \mathrm{~g}$ $\mathrm{H}_{2} \mathrm{O} \mathrm{kg}^{-1} \mathrm{fw}$ ('Carmelo'). Seed dry weight increased by $2.5 \mathrm{~g} \mathrm{(236 \% )}$ from harvest one $(5 \mathrm{maf})$ to harvest three $(7 \mathrm{maf})$, increases which were associated with increments of 0.6 and $4 \mathrm{~mm}$ in seed width and thickness. In seeds recently harvested (high moisture) the germination rate reached $94 \%$, but when seed moisture declined to 300 and $200 \mathrm{~g}$ $\mathrm{H}_{2} \mathrm{O} \mathrm{kg}^{-1} \mathrm{fw}$ the germination rate decreased to $50 \%$ and $0 \%$. The less sensitive genotypes at $300 \mathrm{~g} \mathrm{H}_{2} \mathrm{Ok} \mathrm{g}^{-1} \mathrm{fw}$ were 'EET48' and 'UF667'. Desiccation modified the cell structure of embryo axes. Genotypes 'Carmelo', 'Pound 7', and 'UF668' showed cellular damages (cytoplasm wrinkle, loss of the affinity of nucleic acids to safranin $\mathrm{O}$, and loss of nucleus) in $50 \%$ of the embryo cells at a moisture of 300 and $200 \mathrm{~g}$ $\mathrm{H}_{2} \mathrm{O} \mathrm{kg}^{-1} \mathrm{fw}$. The less sensitive genotype was 'UF668'. It is concluded that cocoa seed sensitivity to drying can be detected among genotypes of the three originis at $300 \mathrm{~g} \mathrm{H}_{2} \mathrm{O} \mathrm{kg}^{-1} \mathrm{fw}$, while with dissection to the level of $200 \mathrm{~g} \mathrm{H}_{2} \mathrm{O} \mathrm{kg}^{-1} \mathrm{fw}$ none of the cocoa genotypes was able to tolerate seed drying.

Index words: Theobroma cacao, moisture content, maturity, level recalcitrant, sensitivity to desiccation.

\section{INTRODUCCIÓN}

Las semillas ortodoxas toleran niveles de desecación hasta de $3 \%$, lo que permite conservarlas por décadas a temperaturas inferiores a $0{ }^{\circ} \mathrm{C}$ en bancos de germoplasma. En contraparte, las semillas recalcitrantes como las de cacao (Theobroma cacao L.), mango (Manguifera indica L.), rambután (Nephelium lappaceum L.) y hule (Hevea brasiliensis L.) (King y Roberts, 1979), no toleran bajos niveles de desecación y por consiguiente se dificulta el resguardo del germoplasma en cuartos fríos, debido a que su alto contenido de humedad las torna sensibles al daño por congelamiento (Roberts, 1973). Esto último demanda la generación de procedimientos que permitan la 
conservación ex situ del germoplasma de estas especies.

Las semillas ortodoxas adquieren la capacidad de tolerar la desecación durante la última etapa de su desarrollo, a través de cambios celulares, fisiológicos y bioquímicos. Entre los cambios celulares, Fisher et al. (1988) encontraron reducción de almidón en los plastidios de los embriones en Sinapis alba L. Por otro lado, Berjak et al. (1992) observaron reducción de vacuolas en embriones de semillas ortodoxas de Landophia kirkii Dyer, mientras que en semillas recalcitrantes de Avicennia marina Forsk no se manifestó esa reducción (Farrant et al., 1997). En semillas de Phaseolus vulgaris L. se reportó una reducción en el número de mitocondrias (Farrant et al., 1997) y alineamiento de cuerpos lipídicos sobre la membrana celular en embriones de maíz (Zea mays L.) (Córdova y Burris, 2002).

El contenido de humedad a la cual la semilla muere, varía entre especies y dentro de la misma especie. En semillas ortodoxas ese contenido fluctúa entre 3 y $7 \%$, mientras que en las recalcitrantes fluctúa entre 12 y $31 \%$ (Wesley-Smith et al., 1992). Chin (1978) reportó que la viabilidad de semillas de alcanfor de Borneo (Dryobalanops aromatica L.), cacao y rambután la viabilidad se redujo considerablemente con humedades de 35, 27 y 20 \%, respectivamente. Además, Dursset et al. (1998) observaron que Coffea arabica es sensible a la desecación con contenidos de humedad inferiores a $8 \%$, mientras que en C. sessiliflora y C. racemosa se observó por debajo de $30 \%$.

Si bien se sabe que existen diferencias en la sensibilidad a la desecación entre especies de un género, como en Coffea (Dursset et al., 1998) y en Dipterocarpus (Tompsett, 1987), poco se conoce de las diferencias entre genotipos de la misma especie. La diversidad genética del cacao cultivado en México está compuesta por germoplasma de orígenes genéticos denominados trinitarios, criollos y forasteros (López et al., 2003). Aun cuando se ha establecido que las semillas de cacao son recalcitrantes, no se ha evaluado la sensibilidad a la desecación de esos orígenes genéticos, lo que podría servir de base para seleccionar genotipos menos sensibles a la desecación, y para elucidar mecanismos genéticos y fisiológicos involucrados en la tolerancia a la desecación al comparar los efectos que ocurren en los menos sensibles con los de mayor sensibilidad. Este conocimiento podría ser de utilidad en la generación de estrategias de conservación del germoplasma ex situ a mediano y largo plazo.

En el presente trabajo se determinó la sensibilidad a la desecación de las semillas de cinco poblaciones de cacao con diferente origen genético, mediante la germinación y el estudio de cambios estructurales en el tejido del eje embrionario. Asimismo, se cuantificaron cambios físicos en la última etapa de desarrollo de la semilla.

\section{MATERIALES Y MÉTODOS}

\section{Material vegetal}

Se emplearon frutos de cinco clones de cacao de tres orígenes genéticos: (1) criollo 'Carmelo', material endémico producto de la selección hecha por los agricultores; (2) forasteros, 'Pound 7' de origen Peruano seleccionado por J. F. POUND, en 1938 y 'EET48' de origen Ecuatoriano seleccionado por EET PICHILINGUE en 1944; y (3) trinitarios, híbridos ‘UF667’ y 'UF668', originarios de Costa Rica seleccionados por UNITED FRUIT CO. Todos los materiales están resguardados actualmente en el banco de germoplasma (colección de campo) del Instituto Nacional de Investigaciones Forestales, Agrícolas y Pecuarias (INIFAP), ubicado en el Centro de Investigación Huimanguillo, Tabasco, en el Km 1 de la carretera Huimanguillo-Cárdenas, Tabasco.

Se cosecharon frutos de los cinco genotipos a los 5, 6 y 7 meses después de la floración (mdf), con base en indicadores de color, tamaño y número de días después de floración. Los frutos se lavaron y desinfectaron con hipoclorito de sodio a $5 \%$ por $1 \mathrm{~h}$; y en seguida se extrajeron las semillas y se lavaron con agua abundante hasta retirar el mucílago.

\section{Cambios físicos de la semilla}

En cada cosecha (5, 6 y $7 \mathrm{mdf})$ y cada genotipo se midieron las dimensiones de la semilla en largo, ancho y espesor, en tres repeticiones de 15 semillas frescas (recién extraídas del fruto). Además, se determinó la humedad y peso seco a $103{ }^{\circ} \mathrm{C}$ por $16 \mathrm{~h}$ (Changrun y Sun, 1999), en tres repeticiones de 15 semillas.

\section{Tolerancia a la desecación}

En cada cosecha, las semillas extraídas del fruto se colocaron sobre toallas de papel a una temperatura entre $20 \mathrm{y}$ $26^{\circ} \mathrm{C}$ y entre 70 y $75 \%$ de humedad relativa, para reducir su humedad a 300, 200 y $150 \mathrm{~g} \mathrm{H}_{2} \mathrm{O} \mathrm{kg}^{-1}$ pf; el testigo fue la humedad de la semilla al momento de cosecha. El daño por secado se evaluó mediante su efecto en la germinación y en la estructura de ejes embrionarios observados después de teñir con safranina y verde fijo. Los tratamientos se distribuyeron en un diseño completamente al azar con arreglo factorial de tratamientos, cuyos factores fueron cosecha, genotipos y niveles de humedad de secado.

Germinación. Se evaluó en las semillas con la humedad que tenían al momento de extracción y en semillas después de secadas a 300, 200 y $150 \mathrm{~g} \mathrm{H}_{2} \mathrm{O} \mathrm{kg}^{-1}$ pf en cada origen, en muestras de 60 semillas repartidas en cuatro repeticiones de 15, colocadas entre toallas de papel de 24 x $23.5 \mathrm{~cm}$ (Marquis Georgia-Pacific) humedecidas con agua destilada. Las toallas se enrollaron en forma de "taco" y se colocaron en una cámara de germinación a $30 \pm 1{ }^{\circ} \mathrm{C}$ (Mumford y Brett, 1982). El primer conteo se efectuó a los 3 d y el segundo a los 7 d después de la siembra; como semillas germinadas se consideró a aquellas con protrusión radicular (King y Roberts, 1982); el valor aquí reportado es la suma de las semillas germinadas en ambos conteos, en porcentaje. 
Estructura del eje embrionario. Con la finalidad de conocer el daño celular en los ejes embrionarios ocasionado por la desecación, se estudió la estructura de las células en cortes anatómicos observados con microscopio de luz. Se extrajeron cinco ejes embrionarios de semillas de los genotipos 'Carmelo, 'Pound 7' y 'UF668', a la cosecha y a 300, 200 y $150 \mathrm{~g} \mathrm{H}_{2} \mathrm{O} \mathrm{kg}^{-1} \mathrm{pf}$, los cuales se fijaron inmediatamente en solución FAA (formaldehído $10 \%$, ácido acético glacial 5 $\%$, etanol $50 \%$ y agua destilada $35 \%$ ) durante $48 \mathrm{~h} \mathrm{a} 4{ }^{\circ} \mathrm{C}$. Este fijador tiene la propiedad de conservar las membranas, las proteínas y los ácidos nucleicos del núcleo (Ruzín, 1999). En seguida las muestras se deshidrataron en una serie de soluciones acuosas de etanol $(30,50,70,80,90$ y $100 \%$, v/v) y se incluyeron en parafina con el procedimiento descrito por Zavaleta-Mancera y Engleman (1991).

Se hicieron cortes $(15 \mu \mathrm{m})$ seriados longitudinales del eje radicular para la reconstrucción de la estructura celular del tejido radicular. Los cortes se desparafinaron y tiñeron con safranina $\mathrm{O}$ (safranina $\mathrm{O} 0.5 \%$ en etanol $50 \%$ ) y verde fijo FCF (verde fijo $0.12 \%$ en etanol 95 \%) (Ruzín, 1999). Las proteínas, membranas y pared celular se tiñeron de verde y los núcleos de rojo, por la afinidad del colorante aniónico (safranina básica) con los ácidos nucleicos, lo que permite reconocer los límites del nucléolo. Como se observaron diferencias visuales en los núcleos asociados con el porcentaje de humedad, se decidió evaluar el daño celular mediante el conteo de núcleos teñidos con safranina $O$ presentes en 50 células de tres repeticiones en tres zonas del eje embrionario (meristemo, caliptra y eje) de los genotipos ('UF 668', 'Pound 7', 'Carmelo'), tanto a la cosecha como a 300, 200 y $150 \mathrm{~g} \mathrm{H}_{2} \mathrm{O} \mathrm{kg}^{-1}$ pf. Las observaciones se efectuaron con objetivos $40 \mathrm{X}$ y $100 \mathrm{X}$ en un microscopio óptico compuesto (Carl Zeiss Axiostar Plus ${ }^{\circledR}$, Germany) y las imágenes se capturaron con una cámara SONY DSC $\mathrm{S} 85{ }^{\circledR}$.

Los datos se analizaron con el paquete estadístico SAS para Windows 6.12, mediante el modelo completamente al azar con arreglo factorial de tratamientos, y la comparación de medias se hizo con la prueba de Tukey $(\alpha=0.05)$.

\section{RESULTADOS}

\section{Cambios físicos de la semilla}

El tamaño, contenido de humedad y peso seco de las semillas cambió $(P \leq 0.05)$ a medida que la cosecha se hizo en un estado más avanzado de madurez, con diferencias entre los genotipos (Cuadro 1). Entre las cosechas el contenido de humedad fluctuó considerablemente, primero con una reducción de $106.9 \mathrm{~g} \mathrm{H}_{2} \mathrm{O} \mathrm{kg}^{-1} \mathrm{pf}$ (15.9 \%) de la cosecha uno a la dos, seguida de un incremento de $46.7 \mathrm{~g} \mathrm{H}_{2} \mathrm{O} \mathrm{kg}^{-1} \mathrm{pf}$ (6.9 $\%)$ de la cosecha dos a la tres. La acumulación en peso seco incrementó $(\mathrm{P} \leq 0.05)$ en $1.2 \mathrm{~g}(163 \%)$ y $2.5 \mathrm{~g}(236 \%)$ en la cosecha dos y tres, respectivamente, en comparación con la cosecha uno. Estos cambios se reflejaron en incrementos de 0.06 y $0.40 \mathrm{~cm}$ en el ancho y espesor de la semilla, respectivamente, de la cosecha uno a la tres, sin cambios significativos en el largo de las mismas.

Respecto a los genotipos (Cuadro 1), los forasteros mostraron mayor reducción de humedad (557.9 $\mathrm{g} \mathrm{H}_{2} \mathrm{O} \mathrm{kg}^{-1} \mathrm{pf}$ ), que representa 75 y $149 \mathrm{~g} \mathrm{H}_{2} \mathrm{O} \mathrm{kg}{ }^{-1} \mathrm{pf}$ menos que los trinitarios y criollo, respectivamente. Los trinitarios acumularon más de $1.09 \mathrm{~g}$ su peso seco e incrementaron en más de 0.24 , 0.11 y $0.13 \mathrm{~cm}$ su largo, ancho y su espesor, en comparación con los forasteros y criollo.

Cuadro 1. Promedios de las variables evaluadas en las semillas de cacao, resultado de los factores principales de variación.

\begin{tabular}{|c|c|c|c|c|c|c|c|}
\hline \multicolumn{3}{|c|}{ Factores } & $\begin{array}{c}\text { Humedad } \\
\left(\mathrm{g} \mathrm{H}_{2} \mathrm{O} \mathrm{kg}^{-1} \mathrm{pf}\right)\end{array}$ & Peso seco (g) & Largo $(\mathrm{cm})$ & Ancho $(\mathrm{cm})$ & Espesor $(\mathrm{cm})$ \\
\hline \multirow[t]{5}{*}{ Cosecha } & (mdf) & & & & & & \\
\hline & & 5 & $672.74 \mathrm{a}$ & $1.83 \mathrm{c}$ & $2.45 \mathrm{a}$ & $0.45 \mathrm{c}$ & $1.35 \mathrm{~b}$ \\
\hline & & 6 & $565.87 \mathrm{c}$ & $2.99 \mathrm{~b}$ & $2.43 \mathrm{a}$ & $0.69 \mathrm{~b}$ & $1.30 \mathrm{~b}$ \\
\hline & & 7 & $612.59 \mathrm{~b}$ & $4.32 \mathrm{a}$ & $2.41 \mathrm{a}$ & $0.85 \mathrm{a}$ & $1.41 \mathrm{a}$ \\
\hline & DHS & & 19.5 & 0.3017 & 0.0791 & 0.0512 & 0.0574 \\
\hline \multicolumn{8}{|l|}{ Genotipo } \\
\hline \multicolumn{8}{|c|}{ Trinitario } \\
\hline & & 'UF668' & $617.36 \mathrm{~b}$ & $3.75 \mathrm{a}$ & $2.60 \mathrm{a}$ & $0.74 \mathrm{a}$ & $1.46 \mathrm{a}$ \\
\hline & & 'UF667' & $645.76 \mathrm{~b}$ & $3.76 \mathrm{a}$ & $2.68 \mathrm{a}$ & $0.79 \mathrm{a}$ & $1.47 \mathrm{a}$ \\
\hline \multicolumn{8}{|c|}{ Forasteros } \\
\hline & & 'Pound 7' & $586.12 \mathrm{c}$ & $2.64 \mathrm{~b}$ & $2.36 \mathrm{~b}$ & $0.63 \mathrm{~b}$ & $1.35 \mathrm{~b}$ \\
\hline & & 'EET48' & $529.62 \mathrm{~d}$ & $2.69 \mathrm{~b}$ & $2.20 \mathrm{c}$ & $0.56 \mathrm{~b}$ & $1.22 \mathrm{c}$ \\
\hline \multicolumn{8}{|c|}{ Criollo } \\
\hline & & 'Carmelo' & $706.5 \mathrm{a}$ & $2.40 \mathrm{~b}$ & $2.30 \mathrm{c}$ & $0.59 \mathrm{~b}$ & $1.29 \mathrm{c}$ \\
\hline & DHS & & 29.618 & 0.4583 & 0.1201 & 0.0778 & 0.0872 \\
\hline
\end{tabular}

Medias con la misma letra en cada variable y para cada factor son estadísticamente iguales (Tukey, 0.05). mdf= meses después de floración. 
El efecto interactivo genotipo $\mathrm{x}$ cosecha se reflejó en el comportamiento diferencial de los genotipos en función de la cosecha para las variables en estudio (Figura 1). El contenido de humedad disminuyó $(\mathrm{P} \leq 0.05)$ en la cosecha dos, con ligero incremento en la cosecha tres en todos los genotipos, pero ninguno alcanzó niveles inferiores a $520 \mathrm{~g}$ $\mathrm{H}_{2} \mathrm{O} \mathrm{kg}{ }^{-1}$ pf. El genotipo 'EET48' exhibió el menor contenido promedio de humedad ( $529 \mathrm{~g} \mathrm{H}_{2} \mathrm{O} \mathrm{kg}^{-1} \mathrm{pf}$ ) en las tres cosechas, mientras que 'Carmelo' se mantuvo en más de $700 \mathrm{~g}$ $\mathrm{H}_{2} \mathrm{O} \mathrm{kg} \mathrm{kg}^{-1} \mathrm{pf}$ y los otros genotipos fueron intermedios entre estos dos (Figura 1A). La acumulación de peso seco creció conforme la semilla se cosechó en un estado de madurez más avanzado; los híbridos 'UF667' y 'UF668', sobresalieron por tener la máxima acumulación (3.75 g) durante el periodo de evaluación, mientras que 'Carmelo' (2.40 g) y 'Pound 7' (2.64 g) mostraron la menor tasa de acumulación de peso seco (Figura 1B). La longitud (datos no mostrados) y el ancho de la semilla cambiaron ligeramente en todos los genotipos (Figura 1C), mientras que el espesor incrementó en promedio $0.40 \mathrm{~cm}$ de la cosecha uno a la tres, en la mayoría de genotipos (Figura 1D).

\section{Tolerancia a la desecación}

Germinación. El efecto de la fecha de cosecha se reflejó en mayor germinación (45\%) a los 6 mdf, superior en

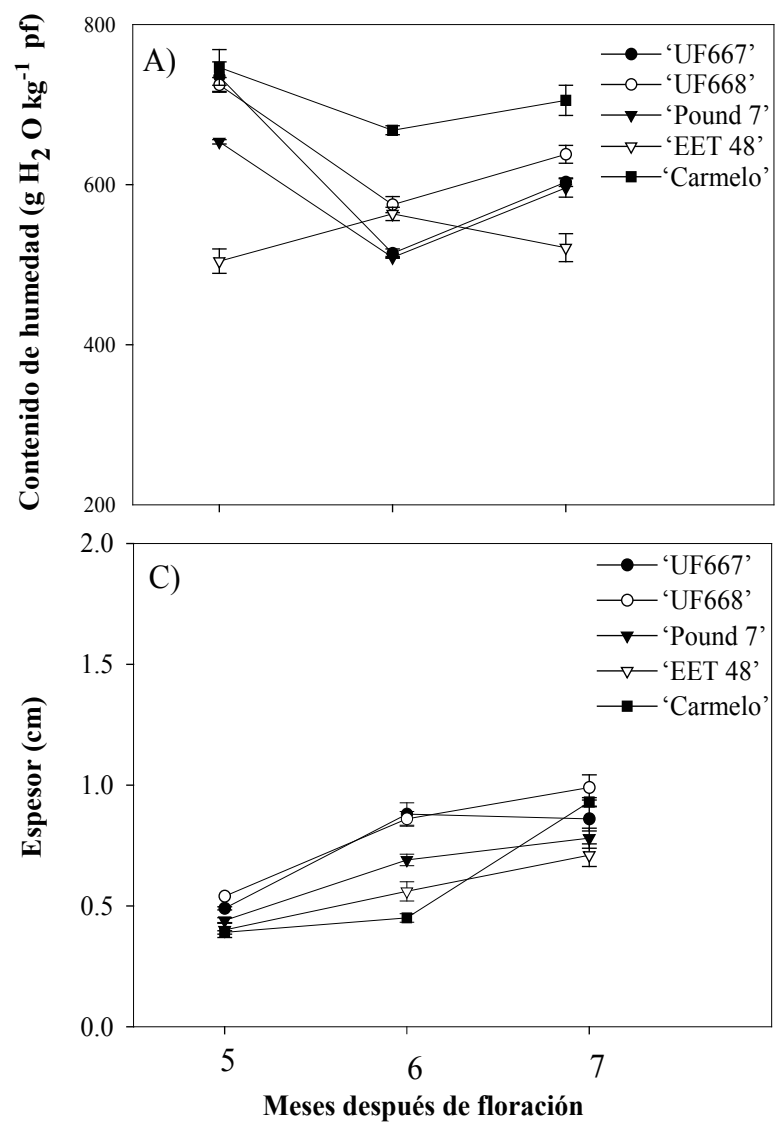

10 y $20 \%$ a la obtenida en la primera y tercera cosecha, respectivamente (Figura 2A). El genotipo 'EET48' tuvo el mayor porcentaje de germinación (41\%), que superó en 19 $\%$ al criollo 'Carmelo' que presentó el menor valor, y en 2, 4 y $5 \%$ a 'UF667', 'F668' y 'Pound 7', respectivamente. El efecto del secado se reflejó en una reducción $(\mathrm{P} \leq 0.05)$ de la germinación y la muerte de la semilla cuando el secado fue inferior a $200 \mathrm{~g} \mathrm{H}_{2} \mathrm{O} \mathrm{kg}^{-1} \mathrm{pf}$ (Figura 2B). Con la humedad de cosecha (617 $\mathrm{g} \mathrm{H}_{2} \mathrm{O} \mathrm{kg} \mathrm{kf}^{-1} \mathrm{pf}$ la germinación promedio fue de $94 \%$, la cual disminuyó en más de $50 \%$ al reducir la humedad de la semilla a $300 \mathrm{~g} \mathrm{H}_{2} \mathrm{O} \mathrm{kg}^{-1}$ pf y en $100 \%$ cuando la humedad se redujo por abajo de $200 \mathrm{~g} \mathrm{H}_{2} \mathrm{O} \mathrm{kg}^{-1}$ pf (Figura 2C).

En la interacción cosecha x genotipos (Figura 2A) resultó evidente el amplió intervalo en germinación que existe en la cosecha uno (5 mdf), que va desde $18 \%$ para 'Carmelo' hasta $50 \%$ para 'EET48'. En la cosecha dos (6 mdf) todos los genotipos alcanzaron como máximo de germinación 50 $\%$, excepto el genotipo 'Carmelo' con $22 \%$. A los 7 mdf (cosecha tres), todos los genotipos redujeron su germinación a $22 \%$.

La interacción cosecha $\mathrm{x}$ nivel de secado (Figura 2B) se debió a que la germinación se redujo conforme la pérdida de humedad fue mayor. En semillas sin secar (humedad
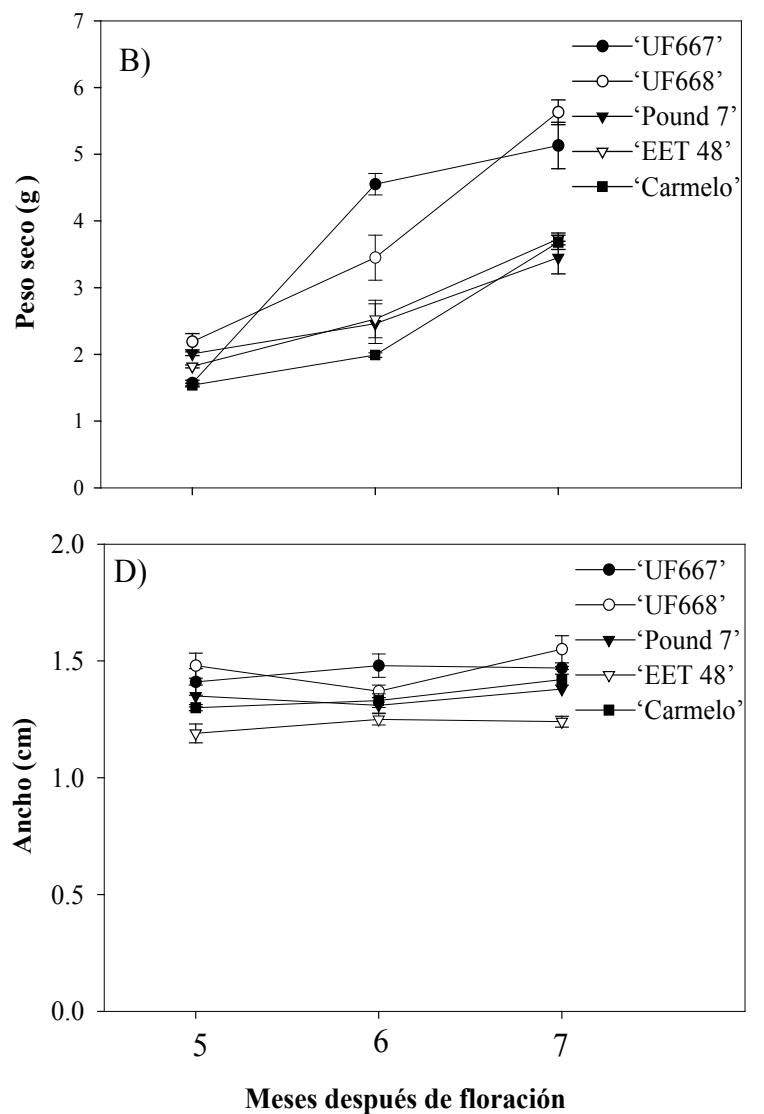

Figura 1. Características físicas de las semillas de cinco genotipos de cacao, cosechadas a los 5, 6 y 7 meses después de floración. A) Contenido de humedad; B) Peso seco, C) Espesor y D) Ancho. 
inicial), la germinación aumentó de 80 a $100 \%$ de la cosecha uno a las cosecha dos y tres. No obstante, al reducir la humedad de la semilla a $300 \mathrm{~g} \mathrm{H}_{2} \mathrm{O} \mathrm{kg}^{-1} \mathrm{pf}$ la germinación disminuyó en más de $20 \%$ en la cosecha uno y dos, y en 100 $\%$ en la cosecha tres.

La interacción genotipos $\mathrm{x}$ niveles de secado (Figura 2C), consistió en que la germinación disminuyó conforme el contenido de humedad fue mayor, pero de manera diferencial entre genotipos. Los genotipos 'Pound 7' y 'EET48' mostraron $100 \%$ de geminación con humedad de cosecha, mientras que los genotipos 'UF667' y 'UF668' solo alcanzaron 90 \% y 'Carmelo' 88 \%. Al reducir la humedad de la
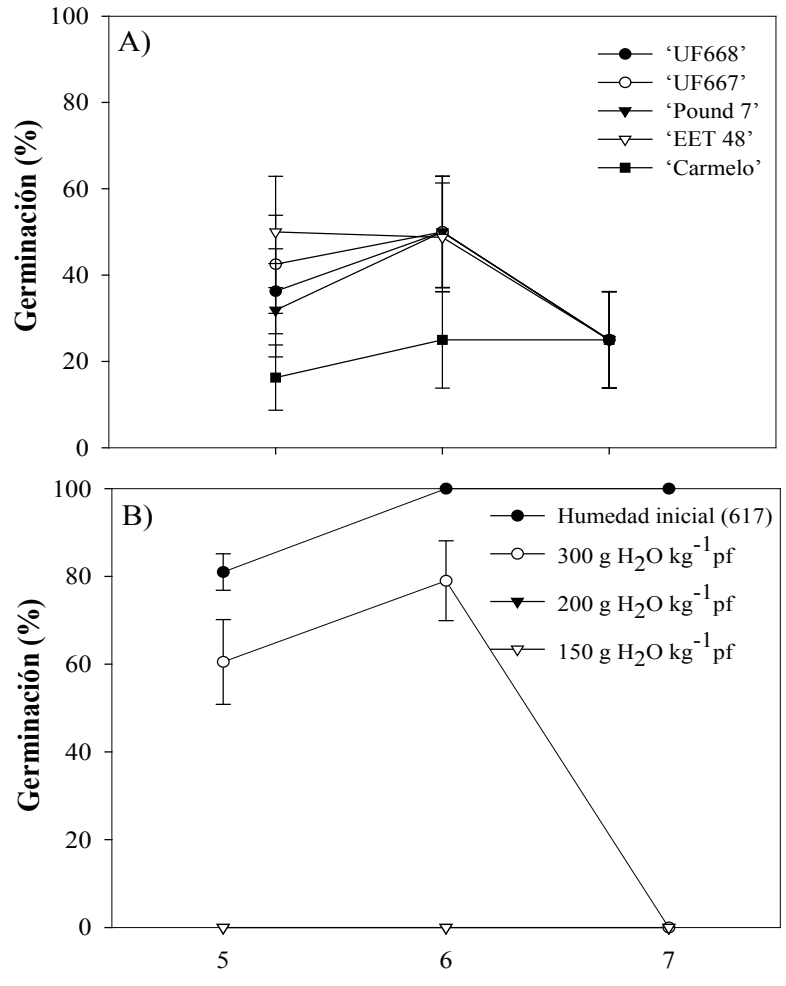

Meses después de floración

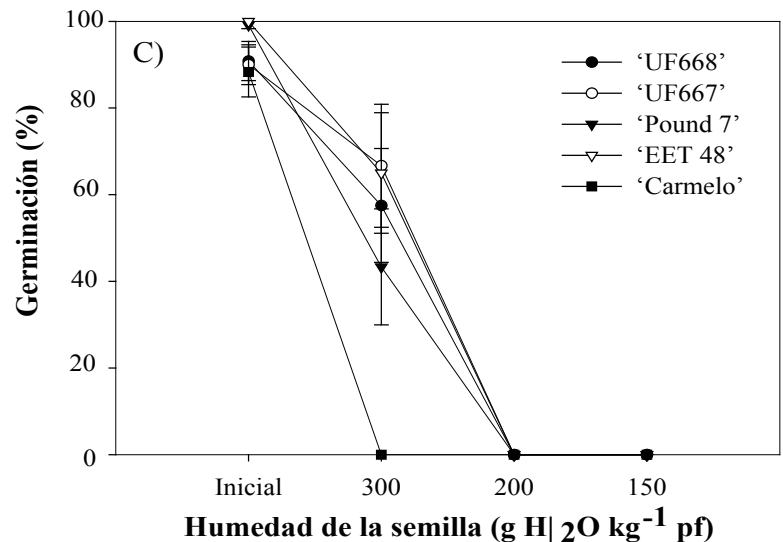

Figura 2. Germinación de las semillas de cinco genotipos de cacao ('UF668', 'UF667', 'Pond7', 'EET48' y 'Carmelo') cosechados a los 5, 6 y 7 meses después de floración (A) y sometidos a tres niveles de secado (B), y comportamiento de los cinco genotipos a los tres niveles de secado (C). semilla a $300 \mathrm{~g} \mathrm{H}_{\mathrm{O}} \mathrm{kg}^{-1} \mathrm{pf}$, 'Carmelo' redujo su germinación en $100 \%$, 'EET48' y 'UF668' en $35 \%$, 'UF667' en $27 \%$ y 'Pound 7' en $57 \%$. Ningún genotipo logro germinar con $200 \mathrm{~g} \mathrm{H}_{2} \mathrm{O} \mathrm{kg}^{-1}$ pf.

Estructura del eje embrionario. Al momento de la cosecha $\left(600 \mathrm{~g} \mathrm{H}_{2} \mathrm{O} \mathrm{kg}{ }^{-1} \mathrm{pf}\right)$, en los tres genotipos el tejido del eje embrionario se caracterizó por la presencia de células meristemáticas turgentes, típicamente isodiamétricas, de pared delgada, sin espacios intercelulares, núcleos grandes y redondeados que ocupaban más de $50 \%$ del volumen
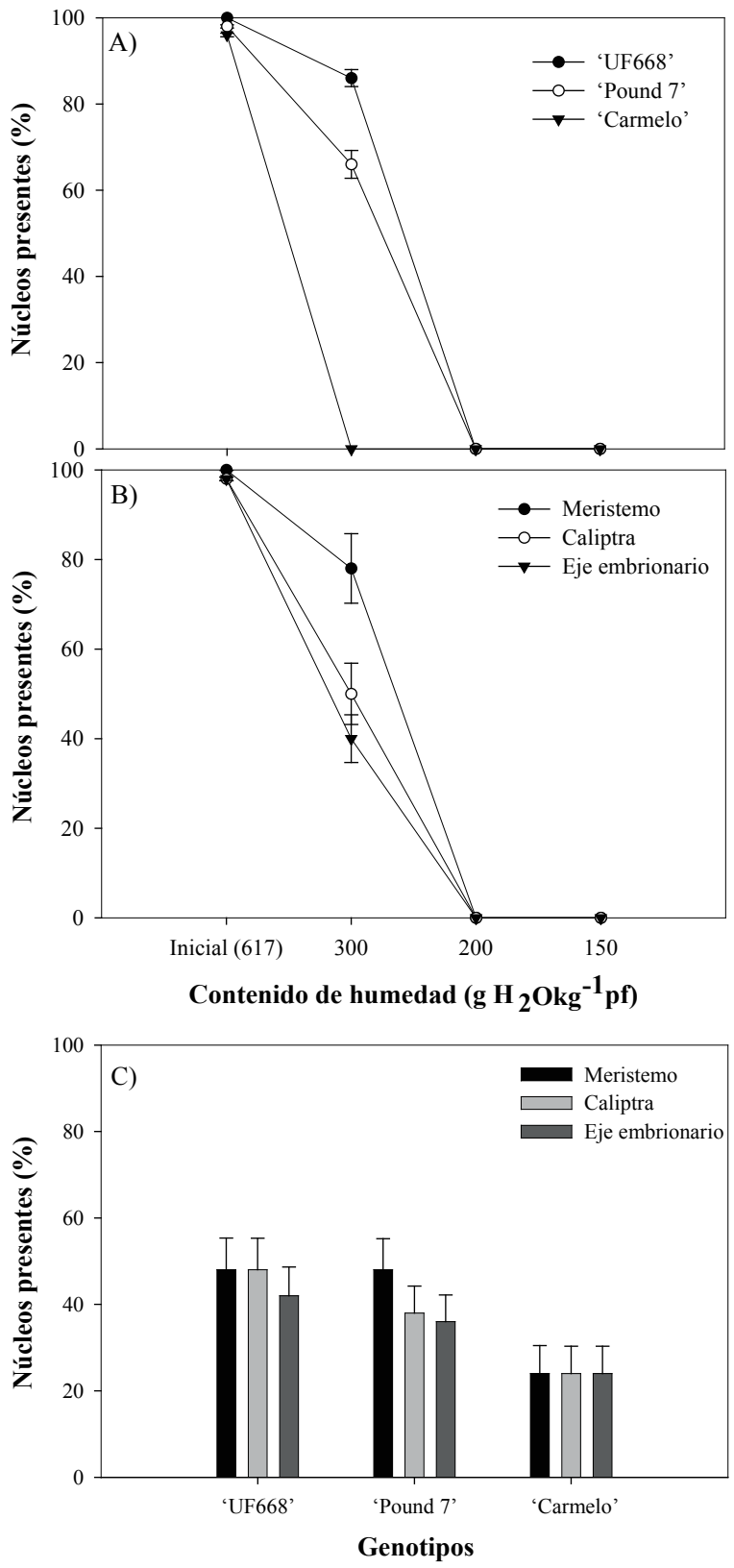

Figura 3. Porcentaje de núcleos en células de tres genotipos de cacao en tres zonas del eje embrionario. A) Núcleos en tres genotipos con diferentes contenidos de humedad; B) Núcleos en tres zonas del eje embrionario a diferentes contenidos de humedad; C) Núcleos en tres zonas del eje embrionario. 
celular; estos núcleos mostraron afinidad por la safranina $\mathrm{O}$ y se tiñeron intensamente de color rojo carmín (Figura 4A). El meristemo apical se observó intacto con células más pequeñas y de forma alargada. La caliptra se caracterizó por la presencia de abundantes taninos. Los principales cambios observados durante la desecación fueron: a) cambios en la afinidad de la safranina por el ADN nuclear, probablemente por su fragmentación; b) aumento en el contenido de granos de almidón; y c) colapso celular.

$\mathrm{Al}$ reducir la humedad a $300 \mathrm{~g} \mathrm{H}_{2} \mathrm{O} \mathrm{kg}^{-1}$ pf los genotipos 'UF668' y 'Pound 7' no presentaron colapso, pero en 14 y $34 \%$ de sus células los núcleos no se tiñeron, y el resto de las células presentaron núcleos de apariencia moteada (fragmentos teñidos en rojo), lo que sugiere cambios en la estructura del ADN (Figura 4B). Cuando la humedad se redujo a $200 \mathrm{~g} \mathrm{H}_{2} \mathrm{O} \mathrm{kg}^{-1} \mathrm{pf}$ (Figura 4C) se detectaron abundantes granos de almidón en las células. En esta condición de desecación, el estudio anatómico de cortes seriados evidenció reducción en el número de núcleos con afinidad por la safranina $O$. En algunas células pudo distinguirse el contorno de la membrana nuclear, pero no en la mayoría (Figura $4 \mathrm{C}$ ). Estas características se asociaron con una reducción de la germinación de 35 \% en 'UF668' y de 57 \% en 'Pound 7 '.
En la condición de $150 \mathrm{~g} \mathrm{H}_{2} \mathrm{O} \mathrm{kg}^{-1}$ pf la desecación se reflejó en reducción del contenido celular con más espacios de aire entre células, señal posible de colapso celular. A pesar de haber efectuado una revisión minuciosa de los cortes seriados, no se detectó presencia de núcleos (Figura 4D).

En la interacción niveles de secado $\mathrm{x}$ genotipo, al reducir la humedad a $300 \mathrm{~g} \mathrm{H}_{2} \mathrm{O} \mathrm{kg}{ }^{-1} \mathrm{pf}$ no fue posible visualizar núcleos en 'Carmelo' (Figura 3A), mientras que en los genotipo 'Pound 7' hubo más de $60 \%$ y en 'UF668' más de $80 \%$. En la interacción niveles de secado x tejidos (Figura 3B), el daño celular se hizo evidente al reducir la humedad a $300 \mathrm{~g} \mathrm{H}_{2} \mathrm{O} \mathrm{kg}^{-1} \mathrm{pf}$, ya que el meristemo radicular perdió $40 \%$ de núcleos visibles. La interacción tejido x genotipo reveló que el criollo 'Carmelo' es más sensible al secado, en eje embrionario, meristemo apical y caliptra, y que 'UF668' fue el genotipo menos sensible (Figura 3C).

\section{DISCUSIÓN}

Las semillas ortodoxas presentan una clara tendencia a reducir su contenido de humedad conforme avanza la etapa de acumulación de materia seca y madurez de la misma (Chandel et al., 1995). En cambio, las semillas recalcitrantes tienden a conservar altos contenidos de humedad, con
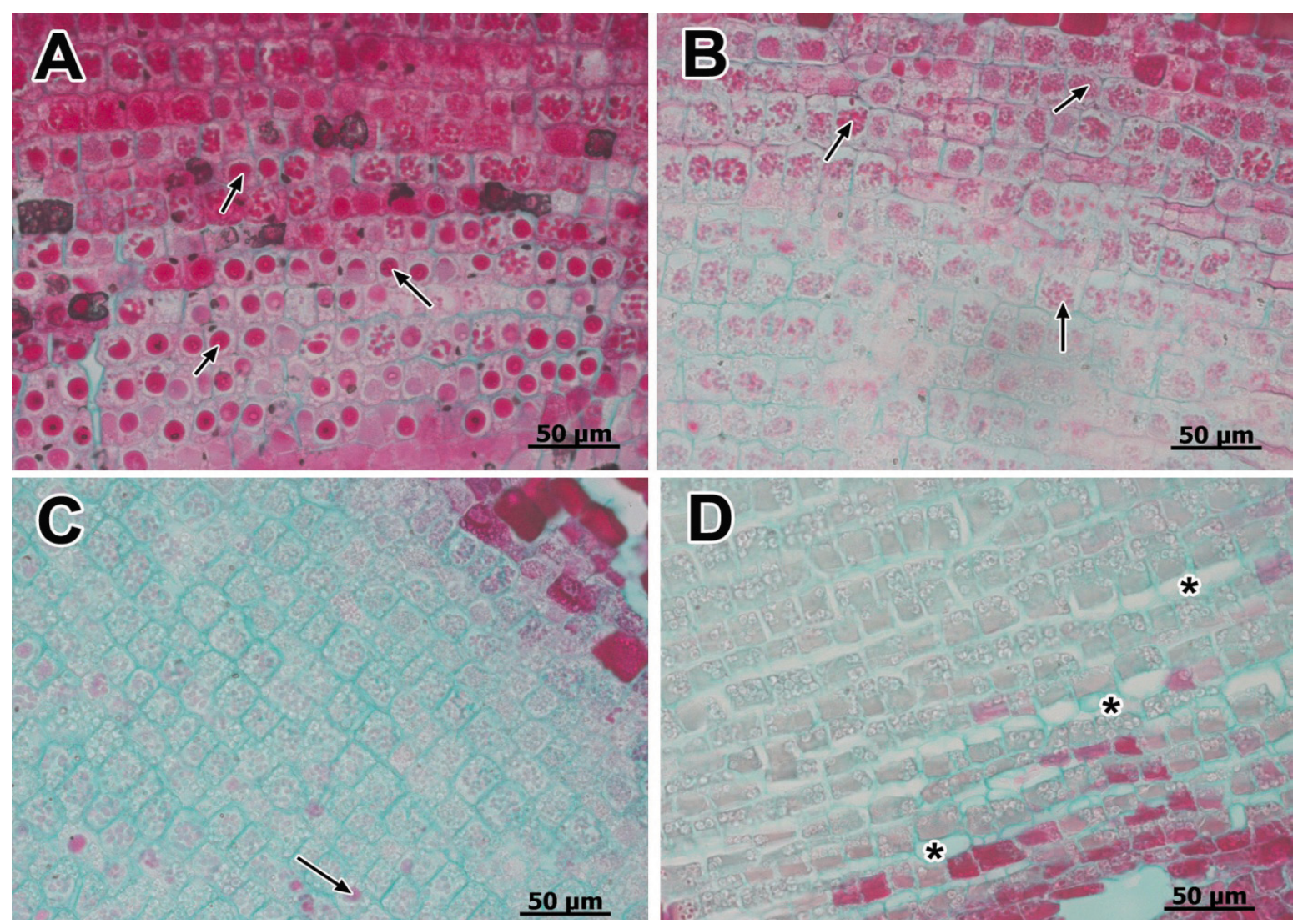

Figura 4. Cambios histológicos en ejes embrionarios de cacao Pound 7: A) con humedad de cosecha $\left.\left(600 \mathrm{gH}_{2} \mathrm{Okg}^{-1} \mathrm{pf}\right), \mathrm{B}\right) 300 \mathrm{gH}_{2} \mathrm{Okg}$ $\left.\left.{ }^{-1} \mathrm{pf}, \mathrm{C}\right) 200 \mathrm{gH}_{2} \mathrm{Okg}^{-1} \mathrm{pf}, \mathrm{D}\right) 150 \mathrm{gH}_{2} \mathrm{Okg}^{-1} \mathrm{pf}$. Las flechas indican núcleos con afinidad a la Safranina; los asteriscos indican los espacios dejados por el colapso celular. 
reducciones mínimas en la última etapa de madurez (Berjak y Pammenter, 2000), comportamiento que coincide con el observado aquí en las semillas de cacao y con lo reportado por Pence (1991) en esta misma especie.

El alto contenido de humedad ( $\left.>520 \mathrm{~g} \mathrm{H}_{2} \mathrm{O} \mathrm{kg} \mathrm{kf}^{-1} \mathrm{p}\right)$ que mantienen las semillas de todos los genotipos a la madurez comercial del fruto (cosecha 3 ), es superior al reportado por Chandel et al. (1995) en semilla madura de cacao (400 a $450 \mathrm{~g} \mathrm{H}_{2} \mathrm{O} \mathrm{kg} \mathrm{kg}^{-1}$ ), pero similar al del eje embrionario (650 a $700 \mathrm{~g} \mathrm{H}_{2} \mathrm{~kg}^{-1} \mathrm{pf}$ ) reportado por los mismos autores en ese estado de madurez. Esa diferencia en valores puede deberse al manejo del fruto y la semilla después de la cosecha (Magnitskiy y Plaza, 2007), pues la semilla pierde humedad rápidamente al exponerse al ambiente una vez que es desprendida de las cubiertas del fruto.

En el presente trabajo es de destacar el reducido contenido de humedad que mantuvo el genotipo 'EET48' con respecto al resto de genotipos, que fue $177 \mathrm{~g} \mathrm{H}_{2} \mathrm{O} \mathrm{kg}^{-1}$ pf inferior al criollo 'Carmelo'. Esto significa que 'EET48' es menos sensible a la deshidratación de las semillas durante la última etapa de desarrollo. Dicha diferencia genotípica podría estar asociada con una mayor eficiencia de 'EET48' en sus sistemas antioxidantes que reducen el daño por secado (Changrun y Sun, 1999), mediante la reducción de radicales libres (Vertucci y Farrant, 1995) que afectan las membranas celulares y viabilidad de la semilla (Tommasi et al., 1999). Según Leprince et al. (1993) la acumulación de grandes cantidades de azúcares solubles y compuestos de reserva contrarrestan los efectos negativos de la desecación, aspectos que fueron corroborados por Rangel-Fajardo et al. (2011).

En todos los genotipos el peso seco de la semilla aumentó con el avance en maduración, pero con valores y tasas superiores en los genotipos del grupo trinitario ('UF667' y 'UF668'), debido posiblemente a que son híbridos y poseen la expresión heterocigótica de genes conocida como heterosis o vigor híbrido. En todos los genotipos el aumento en peso seco se reflejó en incrementos en espesor de la semilla y en menor grado en el ancho, sin cambios significativos en largo ya que esta característica de la semilla queda definida a los 85 d después de polinización (Alemano et al., 1997), fecha alcanzada antes de la primera cosecha efectuado en este estudio.

De la cosecha uno a la dos, en semillas recién cosechadas, la germinación aumentó de 80 a $100 \%$ debido posiblemente a la reducción de embriones inmaduros y latentes por la falta de madurez de la semilla, características que manifiestan varias especies recalcitrantes e intermedias, como en Annona glabra, Quercu ssp. (Mata y MorenoCasasola, 2005; Chávez, 2004). La reducción de humedad a $300 \mathrm{~g} \mathrm{H}_{2} \mathrm{O} \mathrm{kg}{ }^{-1}$ pf provocó la muerte de semillas del criollo 'Carmelo, mientras que los genotipos 'UFF667' y 'EET48' mantuvieron germinación superior a $70 \%$, indicativo de que poseen menor sensibilidad a la desecación. Berjak y Pammenter (2000) señalaron que los niveles de desecación pueden variar entre clones de la misma especie, en concordancia con los resultados del presente estudio.

La desecación a niveles inferiores de $300 \mathrm{~g} \mathrm{H}_{2} \mathrm{O} \mathrm{kg}{ }^{-1} \mathrm{pf}$ provocó mayor daño en las células del eje embrionario, daño que se manifestó por la aparición de espacios intercelulares y ausencia de tinción de núcleos. Esto podría indicar que el contenido de humedad en las células es vital en el mantenimiento de la integridad física de las membranas (Leprince et al., 1993), y en la estructura y funcionamiento del núcleo. Córdova y Burris (2002) observaron colapso celular en el eje embrionario en semillas de maíz previamente sometidas a altas tasas de secado antes de que adquirieran tolerancia a la desecación, etapa que no ocurre en las semillas recalcitrantes como las de cacao y que, en consecuencia, no toleran la deshidratación.

El daño del núcleo causado por desecación se ha reportado en ápices de raíz de lechuga (Lactuca sativa L.), fijados en una solución de ácido acético y alcohol (1:3) y teñidos con aceto-carmín, cuyas células presentaron aberraciones cromosómicas cuando las semillas fueron almacenadas húmedas por períodos mayores a 8 meses (Villiers, 1974). En el presente trabajo, a pesar de una revisión minuciosa de cortes seriados, no se observó evidencia alguna de la

Cuadro 2. Promedios de núcleos (viabilidad celular) en meristemos, cuerpo del eje radicular y caliptra en semillas de cacao de tres genotipos para tres fuentes de variación.

Células con núcleo

Factores

$(\%)$

Genotipo

$\begin{array}{rr}\text { 'UF668' } & 46 \mathrm{a} \\ \text { 'Pound 7' } & 42 \mathrm{~b} \\ \text { 'Carmelo' } & 24 \mathrm{c} \\ \text { DHS } & 0.529\end{array}$

Humedad ( $\left.\mathrm{g} \mathrm{H}_{2} \mathrm{O} \mathrm{kg}^{-1} \mathrm{pf}\right)$

$\begin{array}{rr}\text { Inicial 617 } & 98 \mathrm{a} \\ 300 & 50 \mathrm{~b} \\ 200 & 0 \mathrm{c} \\ 150 & 0 \mathrm{c} \\ \text { DHS } & 0.671\end{array}$

Tejido

$\begin{array}{ll}\text { Meristemo } & 40 \mathrm{a} \\ \text { radicular } & \\ \text { Caliptra } & 38 \mathrm{~b}\end{array}$

Eje embrionario $\quad 34 \mathrm{c}$

DHS

0.529

Medias con la misma letra en cada variable y para cada factor son estadísticamente iguales (Tukey, 0.05). 
presencia de núcleos. Al respecto, Pyung et al. (2007) consideran que el rompimiento de la membrana plasmática conduce al colapso y muerte celular. La mitocondria y el núcleo son los últimos organelos en desaparecer de la célula, ya que el núcleo es el organelo responsable de coordinar los eventos de una muerte celular programada (Pyung et al., 2007). Masetto et al. (2008) detectaron degradación del ADN al reducir la humedad de las semillas de Eugenia pleurantha O. Berg., especie recalcitrante. En este sentido, los resultados en T. cacao sugieren daño nuclear debido a la desecación. En futuros trabajos se sugiere hacer una prueba de TUNEL, un ensayo in situ que permite ver los extremos $\mathrm{OH} 3$ ' del ADN cuando hay fragmentación; esta prueba podría dar certeza sobre la integridad del ADN, como se hizo en estudios efectuados para monitorear la muerte celular en pétalos senescentes de Petunia, Antirrhinum y Argyranthemum (Langston et al., 2005; Yamada et al., 2006).

\section{CONCLUSIONES}

Las semillas de los cinco genotipos de cacao, de tres orígenes genéticos, son todos recalcitrantes pero diferentes en su sensibilidad a la desecación. El genotipo 'EET48' de origen Ecuatoriano y el híbrido Costarricense 'UFF667' fueron los menos sensibles a niveles de deshidratación de 300 $\mathrm{gH}_{2} \mathrm{Okg}^{-1} \mathrm{pf}$, en el que retuvieron $50 \%$ de su germinación. La pérdida de la viabilidad de la semilla de cacao por desecación observada en todos los genotipos estudiados, estuvo asociada con la pérdida en la afinidad de la safranina $O$ por el ADN nuclear, así como con el aumento en el contenido de granos de almidón y del colapso celular.

\section{BIBLIOGRAFÍA}

Alemano L, M Berthouly, N Michaux-Ferriere (1997) A comparison between Theobroma cacao L. zygotic embryogenesis and somatic embryogenesis from floral explants. In Vitro Cel. Develop. Biol. Plant 33:163-172.

Berjak P, N W Pammenter, C W Vertucci (1992) Homohydrous (recalcitrant) seeds: development statuts, deseccation sensitivity and the state of water in axes of Landolphia kirkii Dyer. Planta 186:249261.

Berjak P, N M Pammenter (2000) What ultrastructure has told us about recalcitrant seeds. Rev. Bras. Fisiol. Veg. 12:22-55.

Chandel S P K, R Chaudhury, J Radhamani, S K Malik (1995) Desiccation and freezing sensitivity in recalcitrant seeds of tea, cocoa and jackfruit. Ann. Bot. 76:443-450.

Changrun L, Q Sun Wendel (1999) Desiccation sensitivity and activities of free radical-scavenging enzymes in recalcitrant Theobroma cacao seeds. Seed Sci. Res. 9:209-217.

Chávez Z F (2004) Desecación de bellotas y su relación con la viabilidad y germinación en nueve especies de encinos mexicanos. Ciencia Ergo Sum 11:177-185.

Chin H F (1978) Production and storage of recalcitrant seeds in the tropics: seed problems. Acta Hort. 83:17-21.

Córdova T L, J S Burris (2002) Alignment of lipid bodies along the plasma membrane during the acquisition of desiccation tolerance in maize seed. Crop Sci. 42:1982-1988.

Dursset S, N Chabrillange, F Engelmann, F Anthony, J Louarn, S Ham- on (1998) Cryopreservation of seeds of four coffee species (Coffea Arabica, C. costatifructa, C. racemosa, and C. sessiliflora): importance of water content and cooling rate. Seed Sci. Res. 8:9:15.

Farrant J M, N M Pammenter, P Berjak, C Walters (1997) Subcellular organization and metabolic activity during the development of seeds that attain different levels of desiccation tolerance. Seed Sci. Res. 7:137-144.

Fisher W, R Bergfeld, R Plachy, P Schopfer (1988) Accumulation of storage materials, precocious germination, and development of desiccation tolerance in mustard (Sinapis alba L.). Bot. Acta 101:344354.

King W M, E H Roberts (1979) The Storage of Recalcitrant Seeds. Achievements and Possible Approaches. IBPGR, Rome. Italy. 96 p.

King W M, E H Roberts (1982) The imbibed storage of cocoa (Theobroma cacao L.) seeds. Seed Sci. Technol. 10:535-540.

Langston B J, S Bai, M L Jones (2005) Increases in ADN fragmentation and induction of a senescence-specific nuclease are delayed during corolla senescence in ethylene-insensitive (etr1-1) transgenic petunias. J. Exp. Bot. 56:15-23.

Leprince O, G A F Hendry, B D McKersie (1993) The mechanisms of desiccation tolerance in developing seeds. Seed Sci. Res. 3:231-246.

López A A P, N V H Delgado, M A Azpeitia, A J López, C J Jiménez, F A Rodríguez, S L Fraire, C R Castañeda (2003) El Cacao en Tabasco: Manejo y Producción. INIFAP. Tabasco. México. 81p.

Magnitskiy S V, A G Plaza (2007) Fisiología de semillas recalcitrantes de árboles tropicales. Agron. Colomb. 25:96-103.

Masetto T E, J M Rocha, A C Davide, E A Amaral Da Silva (2008) Desiccation tolerance and DNA integrity in Eugenia pleurantha O. Berg. (Myrtaceae) seed. Rev. Bras. Sem. 30:175-180.

Mata I D, P Moreno-Casasola (2005) Effect of in situ storage, light, and moisture on the germination of two wetland tropical trees. Aquatic Bot. 83:206-218.

Mumford M P, C A Brett (1982) Conservation of cacao seed. Trop. Agric. 59:306-310.

Pammenter W N, P Berjak (1999) A review of recalcitrant seed physiology in relation to desiccation-tolerance mechanisms. Seed Sci. Res. 9:13-37.

Pence C V (1991) Cryopreservation of immature embryos of Theobroma cacao. Plant Cell Rep. 10:144-147.

Pyung O L, H J Kim, H G Nam (2007) Leaf senescence. Annu. Rev. Plant Biol. 58:115-136.

Rangel-Fajardo A, A Delgado-Alvarado, L Cordova-Tellez, H A Zavaleta-Mancera, A Villegas-Monter, A P Lopez-Andrade, I Vidales-Fernandez (2011) Accumulation of sugars in (Theobroma cacao L.) seeds of three genetic origins and its relationship to desiccation tolerance. Seed Sci. Thecnol. 39:1-11.

Roberts E H (1973) Predicting the storage life of seeds. Seed Sci. Technol. 1:499-514.

Ruzin E S (1999) Plant Microtechnique and Microscopy. Oxford University Press. New York. 321 p.

Tommasi F, C Paciolla, O Arrigoni (1999) The ascorbate system in recalcitrant and orthodox seeds. Physiol. Plant. 105:193-198.

Tompsett P B (1987) Desiccation and storage studies on Dipterocarpus seeds. Ann. Appl. Biol. 110:371-379.

Vertucci C W, J M Farrant (1995) Acquisition of desiccation tolerance.. In: J Kigel, G Galili (ed). Seed Development and Germination. Marcel Dekker, New York. pp:237-271.

Villiers T A (1974) Seed aging: chromosome stability and extended viability of seed stored fully hydrated. Plant Physiol. 53:875-878.

Wesley-Smith J, C W Vertucci, P Berjak, N W Pammenter, J Crane (1992) Cryopreservation of desiccation-sensitive axes of Camellia sinensis in relation to dehydration, freezing rate and the thermal properties of tissue water. J. Plant Physiol. 140:596- 604.

Yamada T, K Ichimura, W G van Doorn (2006) ADN degradation and nuclear degeneration during programmed cell death in petals of Antirrhinum, Argyranthemum, and Petunia. J. Exp. Bot. 57:35433552.

Zavaleta-Mancera H A, E M Engleman (1991) Anatomía de Casimiroa edulis (Rutaceae) "zapote blanco", durante su desarrollo. Bol. Soc. Bot. Méx. 51:53-65. 